ISSN electrónico: 1885-5210

DOI: https://doi.org/10.14201/rmc202016e0712

\title{
LA LECTURA DURANTE LA PANDEMIA DE COVID-19
}

\section{Reading during the COVID-19 Pandemic}

\author{
Elena GUARDIOLA; Josep E BAÑOS \\ Facultad de Medicina. Universitat de Vic - Universitat Central de Catalunya (España). \\ Correo electrónico: josepeladi.banos@uvic.cat
}

Fecha de recepción: 4 de septiembre de 2020

Fecha de aceptación: 7 de septiembre de 2020

Fecha de publicación: 29 de enero de 2021

Desde estas páginas hemos reflexionado anteriormente sobre la influencia que la lectura puede tener sobre nuestra salud ${ }^{1}$; también sobre lo que nos ocurre cuando leemos un libro y el efecto que el hecho de leer puede tener en nuestra vida ${ }^{2}$. En esta línea, un estudio publicado recientemente $^{3}$ ha analizado cómo inciden los hábitos lectores sobre la salud y el bienestar de las personas, partiendo de la idea que considera que la lectura no es sólo una fuente de formación e información, sino que puede aportar al individuo otros valores necesarios para su salud y bienestar. A través de un estudio transversal de tipo muestral a personas que tienen el hábito de leer de manera habitual -mediante una encuesta realizada en 2017 de la que se obtuvieron 1.511 respuestas completas, la mayoría procedentes de España-, los autores concluyen que la lectura sirve también para que muchas personas tengan valores gratificantes que redundan en un mejor estado propio de satisfacción y en relación con los otros. Asimismo, los encuestados respondieron que también utilizan y valoran la capacidad que tiene la lectura en relación con aspectos preventivos y saludables como son paliar la soledad, evitar pensamientos negativos y gestionar mejor las emociones. Así, según este estudio ${ }^{3}$, la lectura no sólo entretiene e informa, sino que puede tener otras propiedades, preventivas $\mathrm{y} / \mathrm{o}$ saludables al mejorar el bienestar de los lectores.

Dentro de este marco, la pandemia de COVID-19 y el confinamiento al que hemos estado sometidos durante semanas nos han llevado a preguntarnos cuál ha sido su efecto sobre los hábitos de lectura, así como si leer durante ese período ha tenido un efecto (¿beneficioso?) sobre nuestra salud.

En el mes de marzo, y ya desde los primeros días de confinamiento, empezaron a publicarse resultados de diversas encuestas en las que se 
consideraba el hábito de lectura. Una primera aproximación, que analizó el contenido que leían los consumidores en las páginas web que consultaban durante la pandemia de covid-19, mostró que se había experimentado un gran incremento en una gran variedad de temas, si bien destacaban -como era lógico esperar- los relacionados con la salud, la enfermedad, el bienestar y los hospitales (+ $137 \%)^{4}$. Otro estudio ${ }^{5}$, dado a conocer el 19 de marzo, sobre el impacto del coronavirus en hábitos y medios de comunicación, señalaba que los medios se hacían en ese momento aún más relevantes en su doble dimensión de información y entretenimiento. La televisión, la prensa en línea, Internet, las redes sociales y la radio eran los medios más usados para informarse sobre el coronavirus y el ranking de credibilidad/confianza en esa información lo lideraba la radio, seguida muy de cerca por la televisión y la prensa en papel y en línea. Este estudio señalaba que, entre las actividades de entretenimiento que se hacían más en el hogar, la previsión era que ver la televisión sería la principal (67\%), seguida de cerca por ver películas y series en plataformas de pago (59\%) y leer (53\%), si bien la lectura mostraba diferencias según la edad: $62 \%$ en personas de 40 años o más y $43 \%$ en menores de 40 años. La lectura figuraba, además, entre los seis primeros temas compartidos en las redes sociales.

Al mismo tiempo, desde distintos ámbitos se animaba a la lectura, a quedarse en casa leyendo. Se hacía hincapié en que el entusiasmo lector de los padres ayudaba a la mejora en la competencia lectora de sus hijos y se recordaba que el hogar es el lugar elegido por el $96,6 \%$ de los lectores. Al estar obligados a quedarse en casa, se podía aprovechar para leer, ya que muchas de las alternativas de ocio no eran posibles durante el confinamiento. Se señalaba también que las bibliotecas familiares son una riqueza "estratégica" para los hogares, con una media global de 229 libros en cada hogar español (sin contar los de texto) ${ }^{6}$. Se destacaban los beneficios de leer libros durante la cuarentena y se hacía énfasis en el valor de la lectura como un factor de equilibrio frente a la "fatiga digital" y a la infoxicación durante ese período. Los efectos de la fatiga digital van desde la dificultad para concentrarse a molestias visuales, depresión, ansiedad y otros problemas psicológicos. En este contexto, la lectura sin interrupciones que ofrece un libro se mostraba como un remedio eficaz para contrarrestar la sobrecarga digital de los días de confinamiento, vinculada al fenómeno FOMO (fear of missing out, miedo a perderse algo), que se acentúa en situaciones como la originada por la covid-19. Para las personas más mayores, también desde el punto de vista de la salud neurológica, la lectura era positiva: cuanto más se lee más conexiones entre neuronas se producen, aumentando la reserva cognitiva del lector, un factor protector frente al deterioro cognitivo y la demencia ${ }^{7}$. Se difundían también otros beneficios de la lectura en esa etapa de confinamiento, nueva para la mayoría. Se hacía hincapié en que la lectura de libros ayuda a desconectar y combatir el aburrimiento, crea vínculos con personas cercanas o que comparten inquietudes, activa la memoria y favorece la capacidad de pensamiento crítico, previene el estrés y combate el insomnio8.

Los primeros datos sobre la lectura procedentes de estudios realizados durante el confinamiento empezaron a difundirse ya a principios de abril. Así, se publicaron los resultados de un estudio de la Federación de Gremios de Editores que se llevó a cabo del 25 de marzo al 3 de abril de 2020 cuyo objetivo fue explorar los hábitos de lectura de los españoles durante el confinamiento y su evolución con respecto a un periodo normal. Se realizaron entrevistas telefónicas a 614 personas mayores de edad residentes en España. El análisis de los datos mostró que más de la mitad de los españoles mayores de 18 años aprovechó para leer durante la primera semana de confinamiento y leer se consideró la 
tercera actividad que más ayudaba a sobrellevar la situación, después de ver la televisión y hablar con amigos o familiares por teléfono. Leer en general ocupó el noveno lugar de las actividades más realizadas durante ese período. El $74 \%$ de los que habían leído algo esos días habían leído libros de ocio (novelas, poesías, ensayos, etc.) y el $62 \%$ de los lectores de libros de ocio afirmó que había dedicado más tiempo a la lectura en esos momentos que el que le dedicaba anteriormente (el tiempo promedio de lectura diaria en minutos pasó de 47 a 71). El aumento en el número de lectores a causa del confinamiento se concentró principalmente entre los más jóvenes y, aunque se redujeron algo las diferencias entre sexos en la proporción de lectores de libros, siguieron siendo la mujeres quienes leían más, así como las personas con estudios universitarios. Aunque se observó un cierto incremento de la lectura en soporte digital, estuvo limitado a los grupos de menor edad y el libro en papel se mantuvo como el soporte principal de lectura en todos los grupos de edad ${ }^{9}$. Diversos medios se hicieron eco de estos resultados ${ }^{10,11}$.

Otro estudio ${ }^{12}$ presentó los resultados a finales de abril y mostraba los datos obtenidos a partir de una encuesta en línea en la que participaron más de 2.000 personas desde que se decretó el confinamiento en los hogares. Según este estudio, los lectores habían aumentado en un $63,8 \%$ el tiempo dedicado a la lectura; el $98,3 \%$ de los lectores afirmaba haber continuado con la actividad durante el confinamiento, de los que el $36,5 \%$ confirmó haber aumentado el tiempo dedicado a la lectura en más de cinco horas semanales. El 56,3 \% había leído a diario, con una exposición de una a tres horas confirmada por el 69,9\% de los lectores encuestados. Entre otros muchos datos analizados, podemos citar que el 38,3\% de los lectores había optado por recuperar las lecturas que tenían pendientes en las estanterías y el 55,4\% afirmaba haber realizado la compra de libros en papel, frente al
43,4 \% que había adquirido libros electrónicos y el $1,2 \%$ audiolibros. Asimismo, destacaba que el $96 \%$ afirmaba que los libros se estaban convirtiendo en una pieza clave para superar la situación de confinamiento ${ }^{12}$.

Casi al mismo tiempo, la Fundación Germán Sánchez Ruipérez publicaba los primeros datos sobre lo que la pandemia estaba provocando en el ocio y la lectura ${ }^{13}$. Hacía hincapié en la mayor presencia de la lectura en esa fase y analizaba cómo se comportaba esa propensión a leer más, según tramos de edad y el nivel de ingresos. Concluía que los más jóvenes y las rentas más altas eran quienes habían incrementado más el tiempo dedicado a la lectura. Constataba también que se había producido un fenómeno mundial de incremento de la lectura de libros en el hogar durante la cuarentena, que se igualaba al incremento del consumo de contenidos culturales. Este proceso, que se observaba internacionalmente, se comportaba de un modo similar en España. Señalaba, a continuación, que la cuestión crucial era determinar si estos fenómenos se esfumarían al acabar la crisis de la epidemia o suponían un cambio estable. Este aspecto ha sido analizado también por otros autores ${ }^{14}$.

Desde la ERI Lectura Universitat de València y la Universidad de Salamanca, con la colaboración de la Università degli Studi di Padova, se llevó a cabo un estudio sobre hábitos lectores durante el confinamiento de la covid-19, con el objetivo de analizar y comprender los cambios en los hábitos de lectura de la población adulta española durante las primeras cuatro semanas del confinamiento debido a la irrupción de esta enfermedad. Los datos se obtuvieron a través de una encuesta en línea, realizada entre el 11 y el 19 de abril de 2020, en la que participaron 4.013 personas mayores de edad residentes en España ${ }^{15}$. El análisis de los datos mostró que, durante las cuatro primeras semanas de confinamiento, los españoles aumentaron el tiempo que 
dedicaban a la lectura, destacando el aumento de la lectura social en el $46,1 \%$ de la población y la de ocio en el $45,3 \%$. La lectura de ocio (cómics, revistas o blogs) se incrementó sobre todo en las dos primeras semanas. También aumentó la lectura de noticias en periódicos, revistas, páginas web o a través de Twitter, así como la lectura social de los medios sociales como Instagram, Facebook o WhatsApp. Sin embargo, la lectura para estudio o trabajo, concretamente de informes, webs o documentos, se mantuvo estable. También en este estudio, los hábitos lectores mostraron diferencias entre mujeres y hombres $y$, en general, las mujeres dedicaron más tiempo a la lectura que los hombres. También se observó un incremento de la lectura en soporte digital en las cuatro semanas evaluadas, en comparación con el período anterior al confinamiento, tanto en la lectura de ocio, como en la de estudio/ trabajo y de noticias (especialmente en las dos últimas). Las mujeres mostraron una mayor predisposición hacia la lectura en soporte papel. Entre las conclusiones de este estudio es interesante destacar que los españoles cambiaron sus hábitos lectores durante el confinamiento, que la evolución de las diferentes tipologías de lectura siguió patrones diferentes a medida que avanzó el confinamiento y que la lectura de ocio aumentó de manera generalizada durante ese período de tiempo ${ }^{15}$.

Estudios realizados por plataformas de lectura digital ${ }^{16}$ han señalado también un incremento lector en este soporte, que ha llegado a duplicarse. El confinamiento ha cambiado los hábitos de lectura en digital; mucha gente se ha animado a leer en dispositivos electrónicos y se ha dedicado a la lectura más tiempo, sobre todo durante el fin de semana. Entre los géneros más leídos en este soporte destacan la novela histórica y los libros de autoayuda. Los datos de estas plataformas indican que las mujeres han leído más que los hombres, también en digital (65\% vs.
$35 \%)$ y la horquilla de edad ha sido $30-45$ años. Los niños también han leído más, en compañía de la familia, durante este periodo de reclusión. Este aumento se ha producido también en otros países ${ }^{17}$. Otros estudios indican, además, que el uso de audiolibros también ha experimentado un importante incremento durante la pandemia ${ }^{18}$.

Hasta aquí nos hemos centrado en estudios que han analizado hábitos lectores. No queremos finalizar, sin embargo, sin mencionar una pieza fundamental: el papel de las bibliotecas y de los bibliotecarios/as durante la pandemia y, muy especialmente, durante las semanas que duró el confinamiento y el estado de alarma. Un análisis detallado de la importante función que han desarrollado y de su contribución durante ese período sería, por sí solo, tema de un amplio artículo $^{19-22}$. Desde aquí, nuestro reconocimiento y agradecimiento.

Leer más es siempre una buena noticia. Sin embargo, que esto se produzca a causa de una pandemia, y del confinamiento que conlleva, da mucho que pensar. Volviendo al inicio de este escrito y a pesar de que no disponemos de estudios que lo corroboren, iojalá la lectura en estos tiempos de pandemia haya influido positivamente sobre nuestra salud, así como sobre nuestra vida y nuestro bienestar $!^{1-3}$, iojalá estos nuevos hábitos lectores permanezcan y no se esfumen al acabar la crisis de la pandemia!. ¡Ojalá! 


\section{LA LECTURA DURANTE LA PANDEMIA DE COVID-19 ELENA GUARDIOLA; JOSEP E BAÑOS}

\section{REFERENCIAS}

1. Guardiola E, Baños JE. La lectura ¿influye en nuestra salud? Rev Med Cine. 2017;13(1):1-2.

2. Guardiola E, Baños JE. Lo que ocurre cuando leemos un libro (y su efecto en nuestra vida). Rev Med Cine. 2018;14(1):1-3.

3. Alonso-Arévalo J, Fernández-Martín CL, Alonso-Vázquez A, Mirón Canelo JA. Beneficios de la lectura sobre el bienestar de las personas. Estudio sobre aspectos preventivos de la lectura. Lisboa: APDIS - Associação Portuguesa de Documentação e Informação de Saúde; 2020.

4. Teads. ¿Qué contenido leen los consumidores durante el covid-19? El barómetro de Teads. Marzo de 2020.

5. Havas Media Group. Estudio impacto del coronavirus en hábitos y medios. 19 de marzo de 2020

6. Fundación Germán Sánchez Ruipérez. Quédate en casa leyendo. Marzo de 2020.

7. Fundación Germán Sánchez Ruipérez. Los beneficios de leer libros durante la cuarentena. Marzo de 2020.

8. Comunidad Baratz. 5 beneficios de la lectura de libros durante la cuarentena. 2 de abril de 2020.

9. La lectura en tiempos de covid-19. Madrid: Conecta Research \& Consulting. CONECTA. Abril de 2020.

10. Arcos A. El tiempo dedicado a la lectura aumenta durante la cuarentena. Magisterio. 19 de mayo de 2020.

11. Ayén X. El índice de lectura de España sube un $4 \%$ durante el confinamiento. La Vanguardia. 4 de mayo de 2020. I
12. Exlibric. Estudio de hábitos de lectura durante el confinamiento. Exlibric. 22 de abril de 2020.

13. Fundación Germán Sánchez Ruipérez. Primeros datos sobre lo que la pandemia está provocando en el ocio y la lectura. Abril de 2020.

14. Juárez V. La lectura en tiempos de pandemia. Uvejota. 30 de abril de 2020 .

15. Salmerón L, coord. ERI-Lectura. Hábitos lectores durante el confinamiento por la covid-19 [Informe ejecutivo]. València: RODERIC. Universitat de València; 2020.

16. Nubico blog. La cuarentena ha cambiado nuestros hábitos de lectura: ahora leemos el doble. 21 de mayo de 2020.

17. Pressman A. E-book reading is booming during the coronavirus pandemic. Fortune, 18 de junio de 2020.

18. Proyecto451. La tendencia por el audiolibro continúa creciendo durante la pandemia. 29 de mayo de 2020.

19. How public libraries are responding to the pandemic. American Libraries Magazine. 9 de abril de 2020.

20. Korbey $\mathrm{H}$. How libraries stretch their capabilities to serve kids during a pandemic. KQED; 31 de julio de 2020.

21. Universo Abierto. Blog de la Biblioteca de Traducción y Documentación de la Universidad de Salamanca. El uso de los servicios bibliotecarios ha aumentado considerablemente durante la pandemia. 2 de agosto de 2020.

22. Wilburn T. Libraries are dealing with new demand for books and services during the pandemic. National Public Radio Inc. (NPR). 16 de junio de 2020. 


\begin{tabular}{|c|c|}
\hline & $\begin{array}{l}\text { Elena Guardiola es doctora en Medicina. Investigadora asociada de la Facul- } \\
\text { tad de Ciencias de la Salud y de la Vida de la Universitat Pompeu Fabra } \\
\text { (2007-2018) y desde } 2019 \text { de la Facultad de Medicina de la Universitat de } \\
\text { Vic-Universitat Central de Catalunya, se ha especializado en información, } \\
\text { documentación y redacción científica, áreas en las que ha impartido nume- } \\
\text { rosos cursos. Su interés por la relación entre la medicina y la literatura se ha } \\
\text { plasmado en la participación en varios proyectos así como en la publicación } \\
\text { de diversos trabajos. }\end{array}$ \\
\hline & $\begin{array}{l}\text { Josep-Eladi Baños. Doctor en Medicina. Ha sido profesor de Farmacología } \\
\text { en la Facultad de Ciencias de la Salud y de la Vida de la Universitat Pompeu } \\
\text { Fabra (2002-2018). Vicerrector de Docencia y Ordenación Académica en } \\
\text { dicha Universidad (2005-2013). Ha dirigido el Grupo de Investigación Edu- } \\
\text { cativa en Ciencias de la Salud de la Universitat Pompeu Fabra (2016-2019). } \\
\text { Ha recibido diversas distinciones a la calidad de la innovación docente de } \\
\text { la Generalitat de Catalunya. Desde } 2019 \text { es Rector de la Universitat de Vic } \\
\text { - Universitat Central de Catalunya. }\end{array}$ \\
\hline
\end{tabular}

